\title{
La Región del Cuello del Caballo. Connotaciones Anátomo-Aplicativas: Una Revisión
}

\author{
The Region of the Neck of the Horse. Anatomic Applicative Connotations: A Review
}

\author{
Abelardo Morales Briceño*; Aniceto Méndez Sánchez \& José Pérez Arévalo*
}

\begin{abstract}
MORALES, B. A.; MÉNDEZ, S. A. \& PÉREZ, A. J. La region del cuello del caballo. Connotaciones anatomo-aplicativas: una revisión. Int. J. Morphol., 32(4):1212-1221, 2014.

RESUMEN: La anatomía topográfica veterinaria es el estudio de las relaciones entre las diferentes partes del organismo y es uno de los métodos de estudio anatómico de los animales, es subsiguiente, al otro tipo de estudio, que es de carácter descriptivo, referido a la forma y estructura orgánica mediante una clasificación de órganos por aparatos y sistemas. La región anatómica del cuello es una importante área anatómica y fisiológica por sus múltiples connotaciones anatomo-clínicas. Se plantea como objetivo una revisión anatómica de la región del cuello del caballo y sus connotaciones anatomo-aplicadas. Se realizó una extensa revisión de la literatura de la anatomía del cuello del caballo así como sus connotaciones anatomo-aplicativas, las cuales se describen detalladamente. En la actualidad la región dorsal del cuello ha sido ampliamente estudiada en los caballos debido a su correlación con el síndrome metabólico equino y obesidad en el caballo. En conclusión la región topográfica del cuello representa anatómicamente una gran significación clínica en el caballo por las estructuras y accidentes anatómicos.
\end{abstract}

\section{PALABRAS CLAVE: Anatomía; Cervical; Cresta; Cuello; Equinos.}

\section{INTRODUCCIÓN}

La anatomía topográfica veterinaria es el estudio de las relaciones entre las diferentes partes del organismo y es uno de los métodos de estudio anatómico de los animales, es subsiguiente, al otro tipo de estudio, que es de carácter descriptivo, en el cuál se describe la forma y estructura orgánica mediante una clasificación de órganos por aparatos y sistemas (Olmedo, 2010). La región anatómica del cuello es una importante área anatómica y fisiológica por sus múltiples connotaciones anatomo-clinicas. El cuello es una región impar, unida a la cabeza y al tronco, limitada anteriormente por la nuca, la parótida, la garganta y posteriormente por la cruz, las espaldas y el pecho (Saraza, 1998). La base ósea del cuello está formada por las siete vertebras cervicales, reunidas entre si por articulaciones inter-vertebrales muy móviles. Sobre aquellas encuentran su inserción numerosos músculos, que constituyen dos regiones secundarias: cervical superior y cervical inferior. La región cervical superior está formada por diez y siete músculos, de los cuales seis están colocados en el plano superficial y once en el profundo (Saraza). En la región cervical inferior se encuentran diez músculos: cinco forman el plano superficial y los otros cin- co componen el profundo. Entre los músculos cervicales de las dos regiones superiores, derecha e izquierda, se encuentra el ligamento cervical, producción amarilla elástica muy potente que por su extremidad anterior se inserta en la nuca y por la posterior sobre las vertebras cervicales y dorsales. La tráquea, el esófago, numerosos vasos arteriales, venosos y linfáticos, así como de nervios que recorren la parte inferior de la región cervical inferior (Saraza). En virtud de esta importante área de estudio se plantea como objetivo una revisión anatómica de la región del cuello del caballo y sus connotaciones anatomo-aplicadas.

Osteología de la Región de Cuello del Caballo. El esqueleto del cuello del caballo está formado por 7 vertebras cervicales (Figs. 1y 5), la primera es el atlas (C1), la segunda el axis (C2), posteriormente las vertebras C3, C4, C5, C6 y C7 (Getty, 1996; Pilliner et al., 2002). En general las vertebras cervicales presentan un arco vertebral, un foramen vertebral y un cuerpo vertebral. Las primeras dos vertebras cervicales son diferentes al resto de las vertebras cervicales. El atlas es un hueso tubular corto, con el desarrollo lateral de

\footnotetext{
* Departamento de Anatomía y Anatomía Patológica Comparadas, Edificio de Sanidad Animal, Campus de Rabanales, Universidad de Córdoba, Córdoba, España.
} 
las denominadas alas del atlas con dos forámenes trasversos, se une a la cabeza mediante los cóndilos del hueso occipital y al axis por la superficie articular caudal (fóvea dentis) y los procesos odontoides del axis. En el caballo el foramen alar y el foramen intervertebral se abren en un orificio se abren en un orificio en común (surco común). El axis es un hueso corto, presenta un importante desarrollo de los procesos espinosos dorsales, está unido al atlas por los procesos odontoides que permiten a la cabeza los movimientos de un lado a otro. La superficie superior de los procesos odontoides es desigual para permitir la fijación de un fuerte ligamento que lo mantiene unido al atlas (Pilliner et al.). Las vertebras cervicales $\mathrm{C} 3-\mathrm{C} 7$, tradicionalmente se denominan $(\mathrm{C} 3, \mathrm{C} 4$, C5, C6 y C7), son huesos que presentan reducción de los procesos espinosos (Pilliner et al.), así como proyecciones laterales de los procesos trasversos en donde se insertan los principales músculos y ligamentos del cuello. Estas se unen entre sí por las superficies articulares craneales y caudales. En el equino en la C4 (cuarta vertebra cervical), la lámina ventral de los procesos trasversos se divide en una cúspide craneal y otra caudal (vertebra tricúspide). La ultima vértebra cervical (C7), presenta un importante desarrollo del proceso espinosos dorsal, esta vertebra se une caudalmente con la primera vertebra torácica (T1), mediante sus superficies articulares, ligamentos y músculos.

Sindesmologia de la Región del Cuello del Caballo. Las articulaciones de las vertebras cervicales son de tipo sinovial y planiformes (artroidea). Están constituidas por facetas planas que permiten movimientos de deslizamiento. La articulación entre la primera vértebra cervical y el hueso occipital (permite la unión del cuello a la cabeza), está compuesta

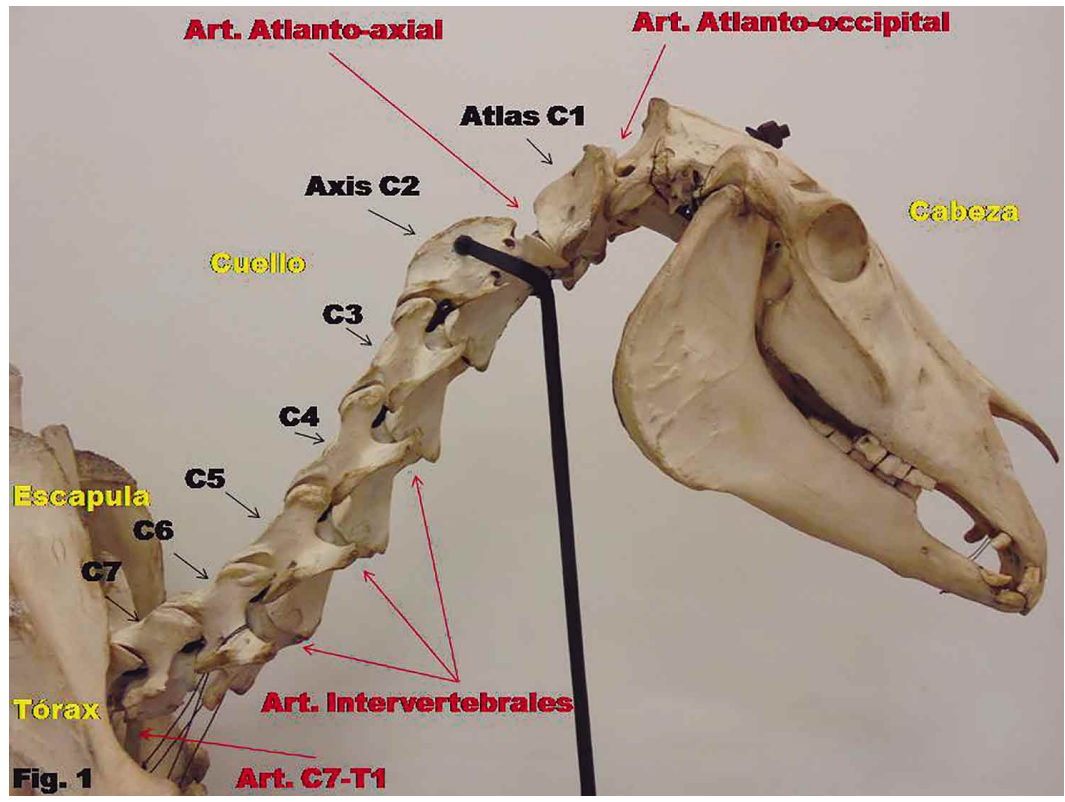

Fig. 1. Base ósea de la región del cuello del caballo (Osteología). por dos articulaciones elipsoidales, en la que se une al respectivo cóndilo occipital del hueso occipital con la correspondiente faceta articular craneal del atlas. Cada una de ambas superficie está rodeada de una capsula articular propia que se inserta en los respectivos bordes articulares (Dyce et al., 2010). En caballos ambas cavidades se comunican entre si pero solo en la avanzada edad. La articulación atlantooccipital es guiada en su función por ligamentos articulares, que como ligamentos laterales pasan sobre el espacio articular entre la cara media de los procesos paracondilares del hueso occipital y la base del ala del atlas (Dyce et al.). Además presenta dos membranas la membrana atlantooccipital dorsal y ventral como refuerzos conjuntivos superficiales de la capsula articular. Los movimientos de la articulación atlantooccipital son de flexión y extensión. La articulación atlantoaxial, es una articulación trocoide, caracterizada por la unión móvil entre los procesos odontoides de la segunda vértebra cervical (axis) y la foseta odontoide del atlas. Esta articulación presenta como ampliación de la superficie articular, las facetas articulares craneales del axis y las facetas articulares caudales del atlas. Todas las superficies articulares están cubiertas por una capsula articular en común, por lo cual se conforma una cavidad articular única. La forma cónica de la superficie articular craneal de los procesos odontoides permite que esta articulación pueda realizar movimientos giratorios desde el eje longitudinal. La capsula articular presenta un refuerzo de la capsula articular caracterizado por colágeno fibroso aportado por la membrana atlantoaxial dorsal entre los arcos vertebrales y por el ligamento axial dorsal, así como ligamentos alares que inician en los procesos odontoides del axis y se insertan en la cara interna del arco vertebral del atlas. El ligamento atlantoaxial ventral constituye un refuerzo en la articulación y vincula el tubérculo ventral del atlas con la cresta ventral del axis (Dyce et al.). El ligamento longitudinal de los procesos odontoides desde la superficie dorsal de los procesos odontoides se ensancha y se inserta en los cóndilos del occipital se encuentra en el canal vertebral de los caballos. Las articulaciones vertebrales cervicales correspondientes a C3, C4, C5, C6 y C7, están unidas por los cuerpos vertebrales, no son articuladas ya que se producen mediante sínfisis intervertebrales con discos intervertebrales que se disponen en la extremidad craneal y ventral (König \& Liebich, 2004). Cada disco intervertebral consta de un núcleo pulposo, que está rodeado por un anillo fibroso, fibrocartilaginoso que está cubierto en su exterior por tejido conjuntivo fibroso. En el caba- 
llo el grosor promedio de los discos intervertebrales es de 2-3 milímetros (König \& Liebich). En el interior del disco intervertebral, el núcleo pulposo en el centro del eje del movimiento de la columna vertebral y presenta una presión interna elevada. Solo están unidas por articulaciones verdaderas los procesos articulares craneales y caudales de los arcos vertebrales que actúan como articulaciones planas (König \& Liebich).

\section{Ligamentos de la Región del Cuello del Caballo}

Los ligamentos vertebrales en general se dividen en cortos que unen solo vertebras vecinas y largos que unen a la columna vertebral en una unidad funcional. Los ligamentos cortos pueden clasificar como: Ligamentos amari-

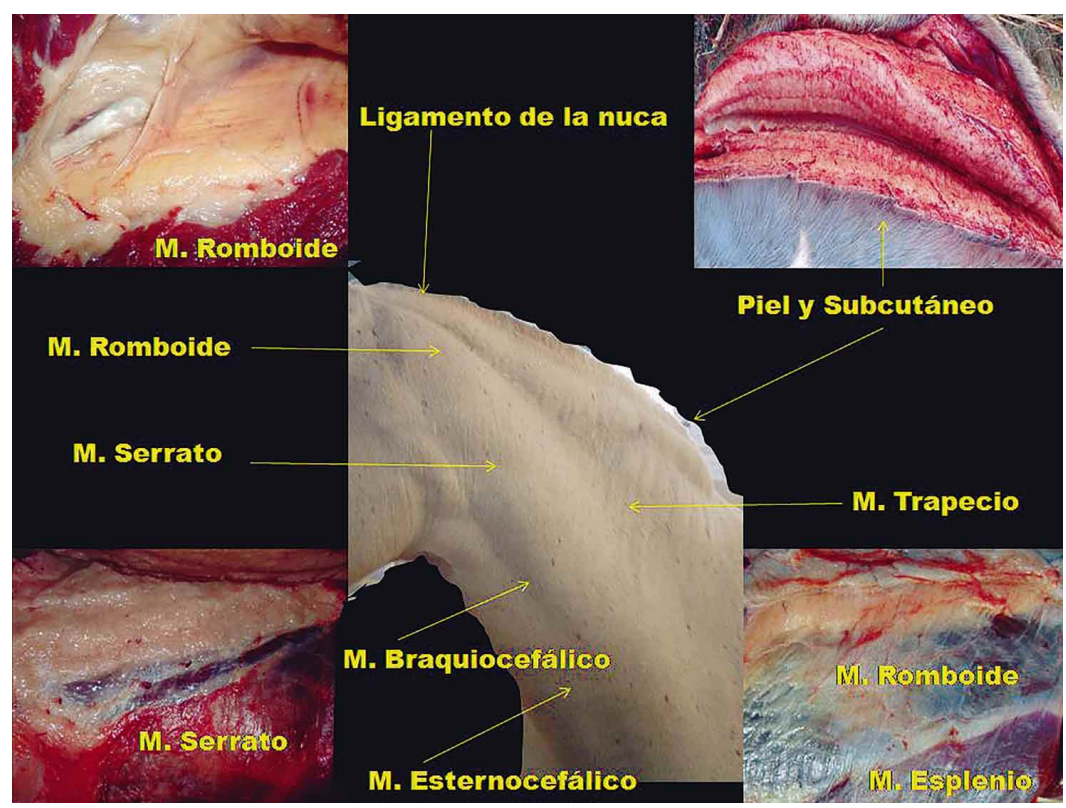

Fig. 2. Base muscular de la región del cuello del caballo (Miología y Sindesmología).

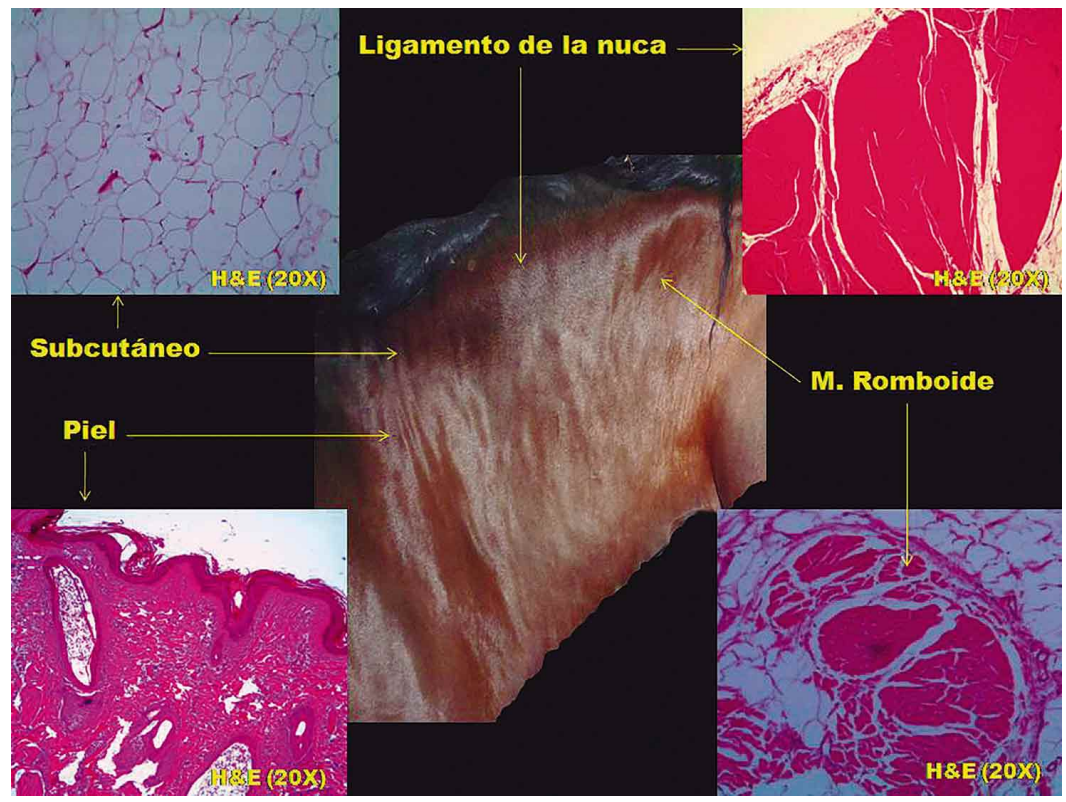

Fig. 3. Base muscular, subcutánea e histológica de la región del cuello del caballo. llos/interarcuales, que cubren los espacios interarcuales como placas elásticas, que se oponen al peso del cuerpo (König \& Liebich). Los ligamentos interespinales se extienden entre los procesos espinosos, son elásticas en la región craneal en el caballo. Estos ligamentos impiden un desplazamiento dorsal de los cuerpos vertebrales y limitan la flexión de la columna vertebral. Los ligamentos intertransversales, entre los procesos transversos poco desarrollados en las vertebras cervicales. Los ligamentos largos se clasifican como: Ligamento longitudinal dorsal, el cual transcurre en el canal vertebral desde los procesos odontoides del axis hasta el hueso sacro, sobre la cara dorsal de los cuerpos vertebrales y se adhiere a las crestas ligamentosas y en los discos inter-vertebrales (König \& Liebich). El ligamento de la nuca que está formado por el funículo de la nuca y la lámina de la nuca, por último el ligamento supraespinoso (Figs. 2 y 3 ). El ligamento de la nuca siempre se encuentra en tenso por el peso de la cabeza y de esta manera disminuye la carga de la musculatura de la cabeza y el cuello. En el caballo este ligamento tiene su origen en la escama occipital, caudalmente se transforma en el ligamento supraespinoso. En el caballo el ligamento de la nuca está formado por el funículo de la nuca y la lamina de la nuca, ambas de desarrollo par. El funículo de la nuca comunica la protuberancia occipital externa, después de ligarse con la lámina de la nuca, a la altura de la tercera vértebra cervical (C3), con los procesos espinosos de la cuarta vertebra torácica (T4) en la que se inserta. En la zona de la cruz el ligamento de la nuca se vuelve ancho para formar el capuchón de la cruz. La lamina de la nuca se inicia en el proceso espinoso del axis y en los tubérculos dorsales de las vertebras cervicales siguientes así como en los procesos espinosos en las ultimas vertebras cervicales y penetra caudalmente en forma de abanico en el funículo de la nuca para finalmente insertarse en el proceso espinoso de la primera vertebra torácica (T1) (König \& Liebich). Debajo de la lamina de la nuca entre el capuchón de la cruz y el proceso espinoso de la segunda (T2) y tercera 
vertebra torácica (T3), se encuentra la bolsa subligamentosa supraespinosa (Bolsa sinovial de la cruz). También en las vertebras cervicales ha sido descrita la bolsa subligamentosa de la nuca craneal del atlas y la bolsa subligamentosa de la nuca caudal del axis.

Miología de la Región del Cuello del Caballo. Debajo de la piel, la superficie de la cabeza y el tronco está envuelta por fascias que permiten el origen e inserción de los músculos y contribuyen a que estos puedan deslizar sobre ella. Estas fascias participan como envoltorio de órganos, además de vasos y nervios en la profundidad. En este punto haremos énfasis en las fascias superficiales del cuello y las fascias profundas del cuello. La fascia superficial del cuello puede estar dividida en una hoja superficial y otra profunda (Figs. 2 y 3 ). La hoja superficial cubre los músculos superficiales del cuello (Músculos: cutáneos, braquiocefálico y el trapecio), el musculo serrato ventral del cuello, musculo esplenio y se inserta en el ligamento de la nuca (König \& Liebich). Esta fascia envuelve a la arteria carótida común (vaina carotidea) y caudalmente continua con la fascia de la espalda y del tronco. La fascia profunda del cuello consta de dos hojas, la hoja superficial que tiene su origen en el ala de atlas, en el musculo largo de la cabeza y en los músculos escalenos. Continúa ventralmente y envuelve al esófago, el nervio laríngeo recurrente, el tronco vagosimpático y la arteria carótida común. Esta fascia se inserta cranealmente en el hueso hioides y en la fascia de la faringe, caudalmente en el primer par de costillas y en el esternón. La hoja profunda nace en los músculos intertransversos y envuelve al musculo largo del cuello y al musculo largo de la cabeza. Los músculos cutáneos junto con las fascias superficiales y profundas, conforman una superficie envolvente de tensión contráctil de particular importancia funcional. El músculo esfínter superficial del cuello se ubica como una estrecha banda muscular trasversal que discurre ventralmente a la laringe por el cuello en dirección caudal. El musculo esfínter profundo del cuello se extiende por debajo de la platisma o del musculo cutáneo de la cara en la superficie lateral del cuello y la cabeza. Este musculo cutáneo tensa la fascia superficial en la región de la laringe. Los músculos cutáneos del cuello reciben su nombre por su posición y función. Son inervados por el ramo del cuello del nervio facial. Los músculos cutáneos del cuello constan de el musculo esfínter superficial del cuello, el platisma, el musculo esfínter profundo del cuello y musculo cutáneo del cuello, el cual es una lámina muscular en la cara ventral del cuello que se origina en el manubrio del esternón y cubre el surco yugular. Los músculos del cuello se encuentran en la nuca y en la cara lateral del cuello, algunos de ellos se asocian con el aparato hioideo (König \& Liebich). El musculo braquiocefálico es el musculo más llamativo y se divide en el musculo cleidobraquial y el musculo cleidocefalico, además del musculo esternocefalico.
El músculo esplenio es fuerte y largo. Está ubicado entre el hueso occipital y la cruz en la región dorsolateral del cuello. Los musculo superficiales del cuello lo cubren lateralmente en forma amplia, a su vez se ubica sobre los músculos largos del cuello. Este musculo se origina en la fascia espinocostotransversa y en el ligamento de la nuca. El músculo esplenio termina como musculo esplenio cervical en los procesos transveros cervicales medias (C3-C5). Como el músculo esplenio de la cabeza se prolonga hasta la cresta de la nuca del hueso occipital y hasta el proceso mastoides del hueso temporal con gran desarrollo y demarcación a través de la piel. El músculo esplenio permite el estiramiento del cuello desde ambos lados así como levanta el cuello y la cabeza. En el salto del galope ayuda a mantener el equilibrio durante el desplazamiento locomotriz. El músculo largo del cuello y los músculos escalenos integran el importante grupo de músculos que inclinan y flexionan la columna cervical. El músculo largo del cuello discurre ventralmente a las vertebras cervicales y vertebras torácicas craneales. En dirección craneal se le adosan, como una prolongación los músculos motores de la cabeza. Su porción torácica tiene su origen en los cuerpos de las primeras quinta a sexta vertebras torácicas (T5-T6) y termina en los procesos transversos de las dos últimas vertebras cervicales (C6-C7). La porción cervical está formada por haces individuales, que nacen ventralmente en los procesos transversos de la tercera a la séptima vértebra cervical (C3-C7). Discurren oblicuamente en dirección craneomedial para insertarse en el plano medio de los cuerpos vertebrales hasta el tuberculoventral del atlas. Los músculos escalenos que discurren desde la primera costilla o de las caras laterales de la octava a la tercera costilla hasta los procesos transversos de la séptima a la tercera vértebra cervical (C3-C7). El plexo braquial divide a los músculos escalenos que se originan en la primera costilla en el músculo escaleno ventral y en el músculo escaleno medio. El músculo escaleno dorsal no se encuentra presente en el caballo. El grupo de músculos hioideos comprende todos los músculos asociados con el aparato hioideo. Los músculos hioideos especiales incluyen: el músculo estilohioideo en el basihioides, el milohioideo, con fibras a través del espacio intermandibular y el músculo genohioideo, que discurre entre el extremo rostromedial del cuerpo de la mandíbula y el basihioides. También pertenecen a este grupo el músculo tirohioideo, el musculo occipitohioideo, el musculo serrato hioideo y el músculo transverso hioideo. Por su unión al basihioides y a la laringe, los músculos largos del hueso hioides refuerzan en forma directa o indirecta el movimiento de la lengua. Representan una parte de los músculos ventrales del cuello y están situados ventrolateralmente a la tráquea. Por lo general, están cubiertos por el músculo braquiocefálico y el músculo esternocefalico y se originan en el manubrio del esternón. Los músculos largos del hioides retraen el aparato hioideo y con ello la lengua. El músculo 


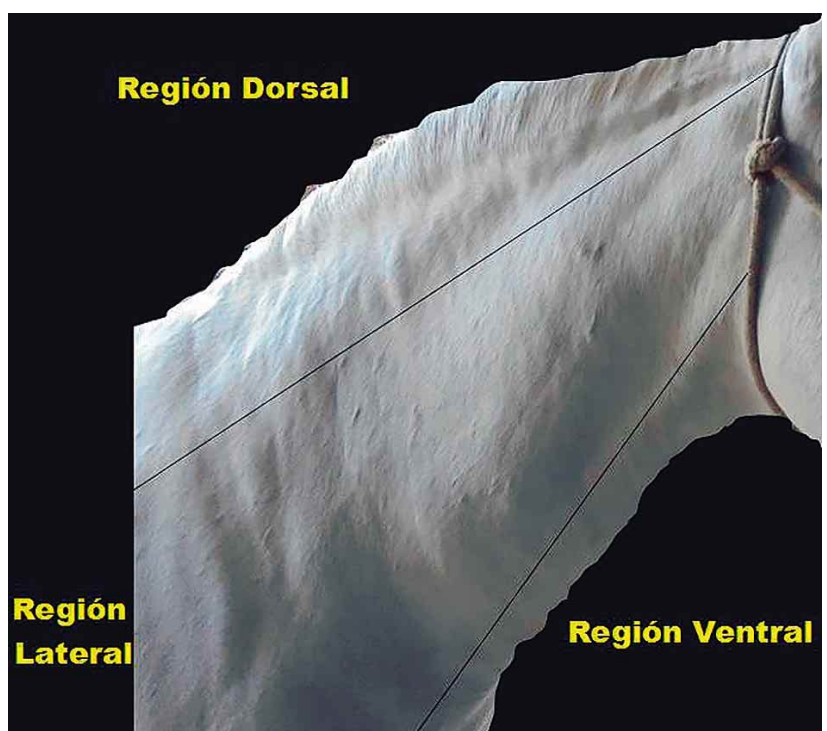

Fig. 4. Regiones anatómicas del cuello del caballo.

esternohioideo tiene un cordón múscular relativamente fuerte que se adosa ventralmente a la tráquea. Se origina en el manubrio del esternón y en la línea media se une al músculo homónimo del otro lado, al músculo esternotiroideo y finaliza en el tirohioides. El músculo esternotiroideo se separa del músculo esternohioideo en la mitad del cuello y se inserta en el cartílago tiroides. El músculo omohioideo alcanza el mayor desarrollo en el caballo. Este se origina en la fascia subescapular cerca de la articulación del hombro y se inserta en el cuerpo del basihioides. Este músculo se une con su homónimo del otro lado al centro del cuello y ambos se insertan junto con el músculo esternohioideo en la proceso lingual del hueso basihioides. En la región superior del cuello ocupa su lugar entre la vena yugular externa y la arteria carótida común. Los músculos dorsales que se adosan a la columna cervical se originan en los cuerpos de sus vertebras o en sus procesos. Estos músculos se pueden dividir en dos grupos: los músculos superficiales y músculos profundos. El grupo múscular superficial se adosa lateralmente al tronco y de acuerdo con su origen embrionario, esta inervado por las ramas ventrales de los nervios del segmento correspondiente. Estos músculos corresponden al musculo trapecio, esternocleidomastoideo, esternocefálico, braquiocefálico, omotransverso, dorsal ancho, pectorales superficiales y serratos dorsales. El grupo muscular profundo tiene su origen embrionario en la somitas de cada localización. Este grupo muscular es conformado por una cantidad numerosa de músculos cortos y largos que al accionarse de forma sinérgica posibilita el movimiento de la columna vertebral. Así como desde el punto de vista funcional los músculos pertenecientes a este grupo también se conocen anatómicamente como los músculos motores especiales de la columna vertebral cervical, torácica y lumbar (König \& Liebich). Por su posición y el trayecto de sus porciones se pueden subdividir en músculos cervicales y músculos dorsales cortos y largos. Los músculos profundos del cuello se presentan como dos cordones musculares alargados y fuertes que se clasifican en un sistema lateral y un sistema medial. Estos sistemas musculares están ubicados en ángulos laterales, entre los procesos espinosos y transversas de las vertebras cervicales. Este sistema es complementado por los músculos: transversos espinales, los interespinales y los intertransversos que son los principales representantes de los huesos cortos del cuello. Los músculos del sistema lateral, consta de segmentos encadenados y fusionados uno tras otro, que en conjunto forman vientres musculares alargados. En la región del cuello, los músculos del sistema lateral están cubiertos superficialmente entre la cruz y el hueso occipital por diferentes planos de la musculatura cervical. Estos músculos se originan en el hueso sacro y en el ilion, así como lateralmente en las vertebras torácicas, en general estos se insertan en las costillas y en la cabeza. El sistema lateral está conformado por los músculos: iliocostal lumbar, iliocostal del tórax, longisimo lumbar, longisimo del tórax, longisimo del cuello, longisimo del atlas y longisimo de la cabeza (König \& Liebich). El músculo iliocostal es un músculo estrecho y alargado, formado por haces de fibras musculares individuales. Sus tendones son segmentarios. Este musculo se origina en cresta iliaca del hueso ilion y en los procesos transversos de las vertebras lumbares y en una placa tendinosa común con el musculo longisimo dorsal y se inserta en la última vértebra cervical (C7). El músculo iliocostal lumbar su porción lumbar se encuentra fusionada con el tendón del musculo longisimo. El músculo iliocostal del tórax se insertan en el borde posterior de la costilla decimoquinta a la primera y a los procesos transversos cervicales. El musculo longisimo (largo del dorso, cuello y cabeza). Este músculo es el más largo del cuerpo ya que se extiende desde los huesos sacros e ilion por todo el dorso y el cuello hasta la cabeza. El músculo longisimo lumbar y el musculo longisimo del tórax tienen su origen en la pelvis y se insertan en la última vértebra cervical (C7). En la región cervical se extienden como músculo longisimo del cuello en forma de abanico desde los procesos transversos de las primeras cinco a ocho vertebras torácicas hasta las últimas vertebras cervicales. Se continúan cranealmente en el músculo longisimo del atlas y el músculo longisimo de la cabeza. Sus tendones se originan en los procesos transversos de las vertebras torácicas segunda y tercera (T2-T3) y de las cuatro a cinco últimas vertebras cervicales y se insertan en el ala del atlas o en el proceso mastoide del hueso temporal. Los músculos del sistema medial forman el plano profundo de la musculatura del cuello y del dorso (König \& Liebich). Los músculos del sistema medial discurren de procesos espinosos (sistema espinal), o bien lo hacen en dirección oblicuo-craneal, desde los procesos transversos hacia los procesos espinosos (sistema transversoespinal). El sistema medial desde el punto de 


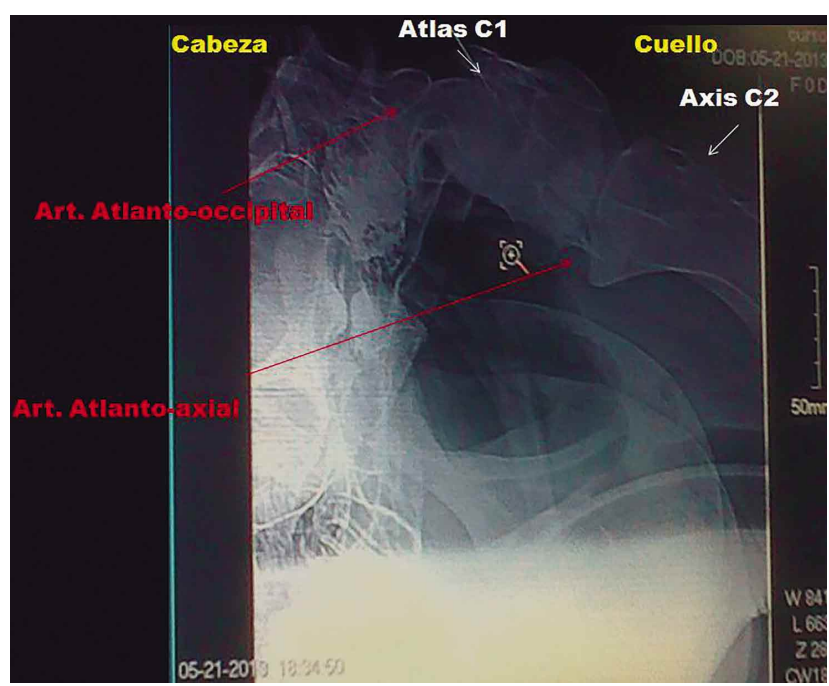

Fig. 5. Radiografía de la región cervical, articulación occipitoatloidea y primeras vertebras cervicales. Cortesía del Dr. Raúl Naranjo Blanco EQCOVET (Guadalajara, México).

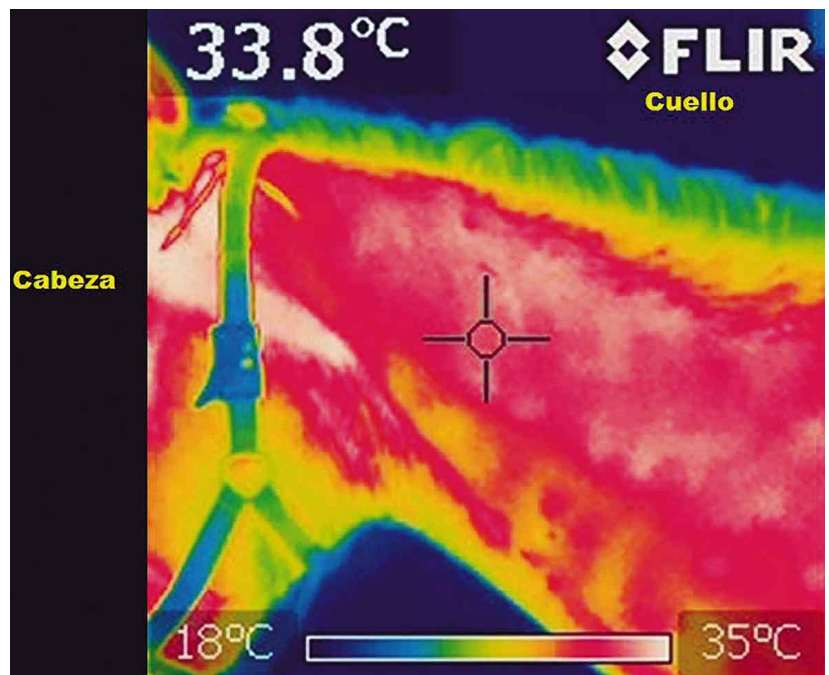

Fig. 6. Termografía de la región del cuello del caballo (Condiciones $19.1^{\circ} \mathrm{C}$ y $64 \%$ de humedad. Cortesía CEDEME, Facultad de Veterinaria UCO (Córdoba, España).

vista funcional se divide en los siguientes músculos: músculo espinal (en sus porciones: del tórax (torácico) y del cuello (cervical); músculos transversoespinales en sus porciones: semiespinal del tórax, semiespinal del cuello, semiespinal de la cabeza (en sus porciones digástrico del cuello y complejo). Los músculos multifidos y los músculos rotadores. El músculo espinal se origina en los procesos espinosos de las vertebras lumbares sexta a la primera y en la de las últimas seis vertebras torácicas y se inserta entre los procesos espinosos de las vertebras torácicas sexta a la primera y medialmente a las vertebras cervicales de la séptima a la tercera (C7-C3) (König \& Liebich). Este musculo discurre exclusivamente entre los procesos espinosos, a menudo saltando varios segmentos, como una sola lamina el músculo espinal del tórax y el músculo espinal del cuello. Cuando este grupo de músculos se contrae de un lado solo, el dorso y el cuello se desvían hacia un costado. El músculo semiespinal de la cabeza, se considera una prolongación del músculo espinal y semiespinal del tórax y cuello, hacia la región de la nuca y hacia la cabeza al que se fusionan lateralmente con el músculo longísimo y el esplenio. Esta importante masa muscular rellena el espacio existente entre el hueso occipital, la columna cervical y el ligamento de la nuca. Por su ubicación se puede distinguir una porción dorsomedial, el músculo digástrico del cuello y otra ventrolateral el musculo complejo. La contracción bilateral del musculo semiespinal de la cabeza determina la elevación de la cabeza. Mientras que la unilateral puede llevar el cuello y la cabeza hacia un lado. Los músculos multifidos, constituyen el plano más profundo de los músculos largos del cuello y del dorso, están formados segmentos musculares que discurren desde los procesos articulares y mamilares. Discurren en dirección dorsocraneal, desde la región lumbar hacia la región del cuello. La función de estos músculos consiste en afinar la secuencia de los movimientos de la musculatura larga del cuello y del dorso. Los músculos rotatorios, pueden ser clasificados como músculos cortos de la musculatura del cuello y la cabeza. Se encuentran en las vertebras torácicas (V1-V16) y permiten los movimientos rotatorios. Los sistemas lateral y medial de los músculos largos del cuello y del dorso, son complementados por desdoblamientos cortos e intersegmentarios (König \& Liebich). Este grupo discurre en los procesos transversas de las vertebras (sistema intertransverso) o entre sus procesos espinosos (sistema interespinal). Los músculos interespinales discurren entre los procesos espinosos desde las vertebras caudales de la columna cervical hasta las primeras vertebras lumbares, bajo la forma de los ligamentos interespinales. Los músculos intertransversos discurren entre los procesos transversos o entre estas y los procesos articulares o bien entre los procesos mamilares y los transversas. En la región cervical los músculos intertransversos dorsales y ventrales del cuello intercomunican los procesos transversos. Este grupo de músculos participa en la sincronización fina de los movimientos de la columna vertebral y ayuda a fijarla o a lateralizarla (König \& Liebich).

Irrigación de la Región del Cuello del Caballo. La irrigación de las estructuras de la cabeza y cuello es provista por las ramas de los vasos sanguíneos que recorren el cuello las cuales se conforman por: Arteria Carótida Común, Vena Yugular Externa, Arteria y Vena Vertebral, Arteria y Vena Cervical Profunda, Plexos Venosos Vertebrales y Arteria Espinal Ventral (König \& Liebich).

Linfonodos de la Región del Cuello del Caballo. En la región cervical del caballo se describe el nódulo linfático cervical superficial (König \& Liebich). 
Inervación de la Región del Cuello del Caballo. En el canal medular de las vertebras cervicales se dispone la medula espinal en segmento cervical, la cual está recubierta por la duramadre, piamadre y aracnoides. Los nervios espinales contienen fibras motoras, sensitivas y autónomas, a nivel cervical presenta 8 segmentos, los cuales llevan el nombre del canal intervertebral por el cual emergen. A nivel cervical se describen dos plexos de nervios cervicales: el ganglio cervical superior, Nervio Vago (Nervio Gran Simpático del Cuello) y el nervio espinal (König \& Liebich). Las áreas de inervación cutánea del cuello en líneas generales, prevalecen en el cuello la organización metamerica o segmentaria que preside su desarrollo y morfogénesis (Sandoval \& Agüera, 1988). Los dermotomos de los primeros segmentos cervicales son mayormente estirados y arrastrados hacia la cabeza y con ellos, los ramos cutáneos de los nervios correspondientes (Sandoval \& Agüera), por ello los dos primeros nervios cervicales contribuyan a inervar la piel de la región occipital y dorso de la oreja con las terminaciones cutáneas de la oreja con las terminaciones cutáneas de los ramos dorsales cervicales $(\mathrm{N}$. suboccipital y N. occipital mayor), por un lado y con los nervios auricular magno y transverso del cuello, por otro, que afectan a la piel de la base lateral de la oreja y regiones masetérica e intermandibular (Sandoval \& Agüera). El área cutánea de los nervios auricular magno y transverso del cuello ocupa plenamente las regiones retroauricular, parotidea y laríngea, y desbordando en mayor o menor proporción sus límites caudales. Los nervios de referencia constituyen la división terminal del ramo ventral del 2 nervio cervical. Prácticamente la región dorsal del cuello y también la mitad caudodorsal de la región lateral quedan ocupadas en superficie por el área cutánea de los ramos dorsales de los nervios cervicales. Los filetes cutáneos de estos ramos, que emergen muy próximos a la línea media dorsal y más lateralmente en el caballo, presentan una uniforme trayectoria caudo-ventral, sobre todo los de niveles caudales, los cuales se manifiestan también más elongados ante el mayor estiramiento de los dermotomos correspondientes (Sandoval \& Agüera). Por idénticas razones de estiramiento el primer dermotomo torácico y su nervio, se incorpora topográficamente a la base dorsal del cuello del caballo. Una dispersión más amplia que las anteriormente descritas corresponde en las tres especies al área cutánea de los ramos ventrales de los nervios cervicales, ocasionada por la ramificación en la piel de los correspondientes ramos de los niveles 3 a 6 y más concretamente, por las derivaciones dorsales y ventrales del Cv3 y Cv4 y por las ventrales del Cv5 y Cv6. El área en cuestión afecta preferentemente a la piel de las regiones braquicefalica, esternocefalica y traqueal. Las derivaciones caudo-dorsales y caudo-ventrales de los ramos ventrales del 6to nervio cervical, emergen la piel de los confines caudoventrales del cuello como nervios supraclaviculares simple- mente o escalonados en nervios supraclaviculares dorsales, intermedios y ventrales. Por consiguiente dichos trayectos nerviosos serán los determinantes de las áreas de los nervios supraclaviculares que afectan a la mitad caudal de la región prescapular y en mayor o menor proporción a las estribaciones caudales de las regiones lateral y ventral del cuello. La disección que muestra estas referencias permite establecer los planos anatómicos precisos en los dos grandes territorios o substratos en que, convencionalmente, dividimos el cuello del caballo para su análisis topográfico, o sea, el sustrato dorso-lateral (región dorsal) y el sustrato ventral (Regiones lateral y ventral). La demarcación vendría dada por la línea que proyecta la serie de procesos transversos cervicales palpables (Sandoval \& Agüera).

Piel y Tejido Subcutáneo de la Región del Cuello del Caballo. El cuello es su superficie está cubierto por piel, subcutis en el borde dorsal, la cresta adiposa de la nuca. El cuello o cresta "Cresty", se redondean y sólo la parte superior de los procesos espinosos son palpables o pueden estar cubiertas de grasa (Figs. 2 y 3). La circunferencia del cuello puede presentar acumulación de grasa alrededor de la zona baja (Henneke et al, 1983; Carroll \& Huntington, 1988). Las medidas del perímetro cervical son importantes para la evaluación de la condición corporal del caballo (Frank et al., 2006; Diez de Castro et al., 2012; Morales Briceño et al., 2014).

Regiones Anatómicas del Cuello del Caballo. Se describen habitualmente tres amplias regiones cervicales dorsal, lateral y ventral (Figs. 4 y 6), así como en otras tres circunscritas, de las que dos (parotidea y laríngea) corresponden a la extremidad cefálica y la tercera a la base del cuello (prescapular); no obstante bajo el punto de vista práctico, se alude a menudo a regiones más concretas, como la braquiocefálica, esternocefalica, retro-auricular, faríngea y traqueal (Sandoval \& Agüera).

Región Dorsal del Cuello. Se prolonga desde la nuca hasta las estribaciones del tórax y la espalda (Regiones Interescapulares y prescapular adyacente). En el caballo se hace progresivamente más extensa a medida que se acerca a la base del cuello, de manera que en el caballo comprende hacia aquí toda la superficie que viene designándose en exterior como "tablas del cuello", también destaca el borde dorsal del cuello que da implantación a las cerdas del cuello o "crinera", el cual está determinado por el cordón de la nuca. Dicho cordón ligamentoso y los músculos adyacentes constituyen la base anatómica de la región (Sandoval \& Agüera).

Región Lateral del Cuello. Es la más extensa de todas las regiones cervicales, puesto que se integra aquí toda la superficie de las tablas del cuello pertenecientes en el caballo, a la región dorsal, además de la región braquicefalica. En el 
caballo si prescindimos del surco yugular que aloja la vena yugular y de la fosa yugular o depresión circunscrita del extremo caudal del surco. La región braquicefalica comprende la mayor parte de la región lateral que nos ocupa (Sandoval \& Agüera).

Región Ventral del Cuello. La constituyen de hecho tres áreas que bajo el punto de vista aplicativo, merecen describirse como regiones más o menos aparentes en la especie que estudiamos. El relieve del músculo esternocefálico designa la región de igual nombre o región esternocefalica, que se prolonga desde el esternón al ángulo de la mandíbula; las dos regiones esternocefalicas (derecha e izquierda), delimitan entre si y perfilan una parte importante de la región traqueal y su continuación o región laríngea, ya en el transito cérvico-facial. Esta última delimita rostralmente con la región sub-hioidea del espacio intermandibular (Sandoval \& Agüera).

Subregiones Ventrales Retroauricular y Parotidea. Ambas regiones se continúan desde la base caudal de la oreja hasta la región laríngea que hemos dejado inscrita en la región ventral del cuello. La primera corresponde al pequeño territorio que queda entre dicha base auricular y el ala del atlas, por lo que su base anatómica mayormente al musculo oblicuo craneal de la cabeza. Ventralmente a ella se prolonga a región parotidea que tiene su base anatómica en la glándula salivar de referencia y un límite craneal muy preciso: el borde caudal de la rama de la mandíbula. Precisamente hacia este límite se advierte en la región bien a simple vista o mediantepalpación una depresión, se trata de la fosa retromandibular que, en la práctica exploratoria, proyecta hacia el espacio intermandibular la región faríngea. Resulta así que la región parotidea, faríngea y la laríngea topografía en conjunto el que denominamos "territorio de transito cérvicofacial", de especial significado anatomo-quirúrgico (Sandoval \& Agüera).

Región Prescapular. Corresponde al límite cérvicoescapular en la base del cuello y más concretamente, al relieve determinado por los músculos subclavio y supra-espinoso, que representan su base anatómica (Sandoval \& Agüera). La región por lo tanto se prolonga desde el ángulo craneal de la escapula hasta la articulación del hombro, pudiendo notar que su límite craneal viene marcado por el surco prescapular, más o menos aparente, ya que sobre los músculos antedichos se superponen las expansiones de inserción en la espina escapular de los congéneres trapecio cervical y del mismo cleidomastoideo.

\section{Accidentes o Referencias de Superficie del Cuello.}

- El surco yugular de la región lateral del cuello del caballo que aloja a la vena yugular externa; lo limita dorsalmente al borde ventral del músculo cleidocefalico y ventralmente, el dorsal del musculo esterno-mandibular; ambos músculos se pueden detectar por palpación a lo largo del cuello.

- La fosa yugular o depresión del extremo caudal del surco, en la que confluye también el surco pectoral lateral; está ocupado por abundante grasa y es área encrucijada vascular, ya que hacia el mismo confluyen, entre otros vasos, las venas tributarias de la yugular externa.

- El surco prescapular ya considerado como limite craneal de la región de igual nombre, que es una referencia precisa en la exploración palpatoria del ganglio linfático cervical superficial (Sandoval \& Agüera).

\section{Los planos topográficos que estratifican los territorios dorso-lateral son:}

\section{Planos Anatómicos Dorso-laterales:}

- Primer Plano: piel y subcutis en el borde dorsal, la cresta adiposa de la nuca.

- Segundo Plano: ramos dorsales cutáneos de los nervios cervicales y la fascia cervical superficial englobando a la porción cervical del músculo trapecio.

- Tercer Plano: cordón de la nuca, músculos romboides y omotransverso y el ramo dorsal del nervio accesorio.

- Cuarto Plano: musculo esplenio y serrato ventral del cuello.

- Quinto Plano: Fascia profunda del cuello, músculos semiespinal de la cabeza y longisimos de la cabeza, atlas y cuello.

- Sexto Plano: lamina de la nuca y los músculos espinal, multifido e intertransverso del cuello.

\section{Planos Anatómicos Ventrales:}

- Primer Plano: piel y subcutis.

- Segundo Plano: fascia cervical superficial, englobando al musculo cutáneo del cuello.

- Tercer Plano: músculos cleidomastoideo y esternomandibular y entre ambos la vena yugular superficial.

- Cuarto Plano: músculos omohioideo y esternotiroideo.

- Quinto Plano: fascia profunda del cuello; en la mitad craneal, el músculo largo de la cabeza hacia el extremo caudal, los músculos iliocostal del cuello y escaleno medio: también la arteria carótida común, el tronco vago simpático y el nervio laríngeo recurrente.

- Sexto Plano: músculo largo del cuello y tráquea por el lado izquierdo, el esófago.

\section{Connotaciones Anatomo-Aplicativas.}

1.- La fascia cervical, coextensible y solidaria con los músculos y otras formaciones del cuello en los movimientos de 
este y de la cabeza (balancín cérvico-cefálico), merece una primera referencia (Sandoval \& Agüera). La lámina superficial cubre la superficie correspondiente de los músculos trapecio, romboides, omotransverso, cutáneo del cuello, cleidomastoideo, esterno-mandibular y omohioideo, además de emitir septos que independizan y empaquetan a los mismos. Los restantes músculos se hacen solidarios con la lamina profunda que los empaqueta de igual modo; concretamente, las partes de esta lamina que cubren el músculo largo del cuello y a la tráquea son las respectivas laminas prevertebral y pretraqueal, siendo la vaina carotidea, la envoltura tubular que protege a la arteria carótida común justamente en el tronco vago simpático y el nervio laríngeo recurrente (Sandoval \& Agüera). Las laminas y vainas de la fascia cervical establecen, así pues, trayectos canaliculares sin solución de continuidad, como, además la fascia profunda tiene su inserción caudal en la primera costilla, esternón y fascia endotorácica.

2.- Las bolsas nucales (sinoviales sub-ligamentosas), que evitan el rozamiento del cordón y cresta adiposa de la nuca con las vertebras contiguas atlas y axis.

3.- Los accidentes palpables de algunas vertebras cervicales y más concretamente los tubérculos dorsales de los procesos transversos C3-C6 y el ala del atlas, proporcionan referencias aplicativas de distinto orden. En lo que respecta a datos de topografía y correlación vertebro-medular debe significarse que dichos tubérculos se proyectan inmediatamente craneales a los agujeros intervertebrales, por lo que también dan cuenta de la situación de los niveles articulares de los correspondientes cuerpos y arcos vertebrales (Sandoval \& Agüera).

Patologías asociadas a la Región del Cuello. En la actualidad el caballo de deporte presenta un número importante de enfermedades que tienen su origen en la región cervical (Diez et al., 2010). Las repercusiones clínicas de las mismas se manifiestan, a grandes rasgos, en diversos grados de ataxia, pérdida de la movilidad del cuello y cojera, entre otros signos (Diez de Castro et al.). Las lesiones del cuello son una causa primaria poco común de dolor, ya sea representado en la claudicaciones o pobre rendimiento atlético, pero se debe considerar si las técnicas analgésicas locales de los miembros no pueden abolir la claudicación o si hay signos clínicos atribuibles directamente al cuello, como el dolor, postura anormal del cue1lo, rigidez o sudoración irregular. El diagnóstico preciso requiere de un cuidadoso examen clínico, la exclusión de otras causas de claudicación o pobre rendimiento, y la interpretación precisa de los resultados de diagnóstico por imágenes (Dyson, 2011). La anatomía detallada de las articulaciones cervicales y de procesos articulares, ha reci- bido poca atención en la literatura, sin embargo estos trastornos se han relacionado con la compresión de la médula espinal que resulta en signos clínicos graves, como la ataxia y la debilidad (Claridge et al., 2010). En un reciente estudio por imagen las anomalías más representativas encontradas en las radiografías fueron la subluxación dorsal oinestabilidad vertebral, la osteoartritis de los procesos articulares y las fracturas vertebrales de distinta naturaleza (Diez et al.). Los diagnósticos más frecuentes obtenidos a partir del estudio radiográfico y clínico fueron la mielopatía estenótica cervical (MEC), y la osteoartritis cervical (OA) (Diez et al.). La región dorsal del cuello es uno de los lugares anatómicos para la deposición de grasa en el caba1lo. El acumulo de grasa en esta región se considera como un signo de obesidad en el caballo (Ralston, 2001). Esta representa una característica física prominente y asociada al síndrome metabólico la adquisición de tejido adiposo en el cuello (Diez de Castro et al.). Las deformaciones del cuello han ido descritas inicialmente como defectos en la cual se han empleado nombres coloquiales. Se distingue el cuello recto o piramidal, las deformaciones del borde superior que se puede observar suavemente arqueado (cuello de gato o de pichón), así como el defecto de ser muy grueso el borde superior del cuello por abundante deposito de grasa provisto de una crinera abundante, y por efecto de la debilidad de la región se muestra algo caído en alguno de sus lados (gato o gatillo vencido hacia la derecha o hacia la izquierda), los cambios en la convexidad del borde superior del cuello y que se acentúa en su punto de unión con la cabeza (cuello de cisne), cuando su borde superior es ligeramente cóncavo y el inferior convexo (cuello al revés o de ciervo) (Saraza). La deformación del borde dorsal del cuello (DBDC), es una condición que tiene gran repercusión clínica y económica (Ruiz López et al., 2010), que se presenta en caballos de Pura Raza Española y en otras razas como la Lusitana. Recientemente un estudio describe la deformación del borde dorsal del cuello en burros de raza Andaluza (Morales Briceño et al.). En conclusión la región topográfica del cuello representa anatómicamente una gran significación clínica en el caballo por las estructuras y accidentes anatómicos que se encuentran en esa área.

\section{AGRADECIMIENTOS}

Los autores agradecen al CEMEDE (Centro de Medicina Deportiva Equina), de la Universidad de Córdoba, España, por la imagen cedida de la termografía de la región cervical de un caballo y al Dr. Raúl Naranjo Blanco (EQCOVET), por la imagen radiográfica cedida de la región cervical del caballo. 
MORALES, B. A.; MÉNDEZ, S. A. \& PÉREZ, A. J. The region of the neck of the horse. Anatomic applicative connotations: a review. Int. J. Morphol., 32(4):1212-1221, 2014.

SUMMARY: Veterinary topographic anatomy is the study of relationships between different parts of the body and is one of the methods of anatomical study of animals; it is subsequent to the other study, which is descriptive based on the shape and structure rated by organic organs organ systems. The anatomical neck region is an important anatomical and physiological area because of its many anatomical and clinical implications. The aim of this study was to a review of an anatomical region of the horse's neck and applied anatomical connotations. An extensive literature review of the anatomy of the horse's neck and its applicative anatomical connotations, which are described in detail, was conducted. Currently the dorsal neck region has been extensively studied in horses due to their correlation with equine metabolic syndrome and obesity in the horse. In conclusion topographic anatomical neck region represents a great clinical significance in the horse by accident and anatomical structures.

\section{KEY WORDS: Anatomy; Cervical; Crest; Neck; Horses.}

\section{REFERENCIAS BIBLIOGRÁFICAS}

Claridge, H. A.; Piercy, R. J.; Parry, A. \& Weller, R. The 3D anatomy of the cervical articular process joints in the horse and their topographical relationship to the spinal cord. Equine Vet. J., 42(8):726-31, 2010.

Carroll, C. L. \& Huntington, P. J. Body condition scoring and weight estimation of horses. Equine Vet. J., 20(1):41-5, 1988.

Diez de Castro, E.; Pineda Martos, C.; Martin Cuervo, M.; Quintero Felices, S.; Mendez Vazquez, N. \& Aguilera Tejero, E. Valoración de obesidad y resistencia a la insulina en el caballo de pura raza española (PRE). Equinus, 34:58-75, 2012.

Díez, E.; Sánchez de Medina, A. \& Novales Durán, M. Aportaciones de la radiología digital al estudio de las patologías cervicales del caballo. Equinus, 27:53-68, 2010.

Dyce, K. M.; Wensing, C. J. G. \& Sack, W. O. Tratado de Anatomia Veterinária. 4a ed. Rio de Janeiro, Elsevier, 2010. pp.532-50.

Dyson, S. J. Lesions of the equine neck resulting in lameness or poor performance. Vet. Clin. North Am. Equine Pract., 27(3):417-37, 2011.

Frank, N.; Elliot S. B.; Brant, L. E. \& Keisler, D. H. Physical characteristics, blood hormone concentrations, and plasma lipid concentrations in obese horses with insulin resistance. J. Am. Vet. Med. Assoc., 228(9):1983-90, 2006.

Getty, R. Anatomía de los Animales Domésticos. 5a ed. México D. F., Salvat Editores, 1996.

Henneke, D. R.; Potter, G. D.; Kreider, J. L. \& Yeates, B. F. Relationship between condition score, physical measurements and body fat percentage in mares. Equine Vet. J., 15(4):371-2, 1983.

König, H. E. \& Liebich, H. G. Anatomía de los animales domésticos. 2a ed. Buenos Aires, Médica Panamericana, 2004. pp.91120.
Morales Briceño, A.; Méndez Sánchez, A.; Pérez Arévalo, J.; Lamprea Garrido, A.; García Hermoso, A. \& Díaz García, M. Estudio clínico morfológico de la deformación del borde dorsal del cuello del burro de raza andaluza. Rev. Complut. Cienc. Vet., 8(1):1-9, 2014.

Olmedo, P. G. Diseño de tareas de aprendizaje para el desarrollo de competencias y pensamiento complejo. Veracruz, Facultad de Ciencias Biológicas y Agropecuarias Región Poza Rica Tuxpan, Universidad Veracruzana, 2010. Disponible en: http:/ /www.uv.mx/personal/golmedo/files/2011/05/Informe-ATVA2010.pdf

Pilliner, S.; Elmhurst, S. \& Davies, Z. The horse in motion. Oxford, Blackwell Science, 2002. pp.14-23.

Ralston, S. L. Maintenance of the "Easy Keeper" Horse. New Jersey, Rutgers Cooperative Extension, New Jersey Agricultural Experiment Station, The State University of New Jersey, 2001. pp.750-1201.

Ruiz López, I.; Armengou, L.; Chamizo, V.; Valdés, M. \& López Rivero, J. Deformación del borde dorsal del cuello en caballos de pura raza española: incidencia y aspectos clínicos. Equinus, 26:24-34. 2010.

Sandoval, J. \& Agüera, E. Anatomía Aplicada Veterinaria: caballo, vaca, perro. 2a ed. Salvat-Editores, 1998. pp.120-80.

Saraza, M. J. Exterior del caballo. Badajoz, Colegio Oficial de Veterinarios de Badajoz, 1998. pp.132-40.

\section{Dirección para Correspondencia: \\ Departamento de Anatomía y \\ Anatomía Patológica Comparadas \\ Edificio de Sanidad Animal, \\ Campus de Rabanales Ctra. de Madrid km 396, \\ 14071 Córdoba \\ Universidad de Córdoba \\ ESPAÑA}

Email: aamorales13@gmail.com

Received: 27-04-2014

Accepted: 04-08-2014 